\title{
Quality of care indicators and their related outcomes: A population- based study in prostate cancer patients treated with radical prostatectomy
}

\author{
Colleen Webber, MSc; ${ }^{*}$ D. Robert Siemens, MD, FRCSC;, ${ }^{+\xi}$ Michael Brundage, MD, MSC, FRCPC; ${ }^{*+}$ \\ Patti A. Groome, PhD*
}

*Division of Cancer Care and Epidemiology, Queen's Cancer Research Institute, and Department of Public Health Sciences, Queen's University, Kingston, ON; 'Department of Oncology, Queen's University, Kingston, ON; §Department of Urology, Queen's University, Kingston, ON

Cite as: Can Urol Assoc J 2014;8(7-8):e572-9. http://dx.doi.org/10.5489/cuaj.1649

Published online August 11, 2014.

\section{Abstract}

Introduction: We evaluated the validity of 8 quality of care indicators for prostate cancer patients treated curatively with radical prostatectomy (RP) by examining their association with indicatorrelevant outcomes.

Methods: We conducted a population-based retrospective cohort study of 646 prostate cancer patients diagnosed between 1990 and 1998 who received RP within 6 months of diagnosis. Data were collected from treating charts and linked to registry and administrative data. Quality indicators included: hospital volume, pre-treatment risk assessment, consultation with a radiation oncologist, appropriate follow-up care, nerve-sparing surgery, units of blood transfused, surgical margin status, and pelvic lymph node dissection during RP. Indicator-relevant outcomes were selected a priori by clinical members of the research team. The associations between indicators and their relevant outcomes were analyzed using regression techniques, to control for potential confounders. Results: Of the quality indicators evaluated, only hospital volume was statistically significantly associated with the gradient in the expected direction. Patients treated in the lowest-volume hospitals $(<1 \mathrm{RP} /$ month) had lower cause-specific survival rates compared to patients treated in the highest-volume hospitals ( $\geq 7 \mathrm{RP} / \mathrm{month}$ ) $(\mathrm{HR}=4.71$ 95\%; Cl 1.06-20.82). Completeness of follow-up care was associated with cause-specific survival but in the opposite direction to our hypothesis.

Conclusion: The structural indicator of hospital volume was associated with cause-specific survival in accordance with our a priori hypothesis. Our negative findings for completeness of follow-up care call its validity into question. Issues of statistical power and measurement accuracy may have affected our validation of the remaining indicators underscoring the challenges in assessing the impact of accepted quality indicators.

\section{Introduction}

Recent information about the considerable variations in the management and clinical outcomes of prostate cancer has led to an increased interest in the evaluation of the quality of care provided to prostate cancer patients. Many stakeholder groups have called for constructive tools to help ensure the best possible care, and several have published quality indicator lists for surgical management. ${ }^{1-3}$ To date, the validity of these indicators has not been well established.

A common approach to measurement validation is to compare the measurement against a "gold standard" (i.e., criterion) measure of the same construct; no such globally accepted measure of quality of care exists, however. Alternatively, we can use the quality of care framework developed by Donabedian to guide our thinking about the validity of quality of care measures. This framework states that quality comprises three components: structure, process, and outcome, and that these components are interrelated such that high quality in one component can lead to high quality in the others. ${ }^{4}$ Thus, we can validate quality indicators by demonstrating associations between structure and process measures of quality and their relevant clinical outcomes.

The goal of this study was to demonstrate that Donabedian's framework can be used as a foundation upon which to base efforts to validate quality of care indicators in prostate cancer management. We examined the validity of 1 structural and 7 process-related quality indicators for prostate cancer patients treated curatively with radical prostatectomy (RP) by examining their associations with indicator-relevant outcomes. The validity of proposed indicators was demonstrated if they were associated with outcomes in accordance with our a priori hypotheses. 


\section{Methods}

We conducted a population-based retrospective cohort study with a study population of surgically treated prostate cancer patients diagnosed in Ontario between 1990 and 1998. Data for the study were collected as part of a larger populationbased case-cohort study designed to evaluate variations in case selection and outcomes across and within treatment modalities in prostate cancer. ${ }^{5} \mathrm{~A}$ linked population-based database containing the Ontario Cancer Registry (OCR), hospital discharge data, and cancer clinic data housed at the Queen's University Cancer Research Institute Division of Cancer Care and Epidemiology $(\mathrm{CCE})^{6}$ was used to identify all curatively treated patients with adenocarcinoma of the prostate diagnosed in Ontario between January 1, 1990, and December 31, 1998. We selected a region-stratified random sample of approximately $10 \%$ of the 17934 patients meeting these criteria $(n=1703)$. After chart review, a further 133 patients were excluded because they were not candidates for curative treatment, they received only lymph node dissection or no treatment, they received surgery more than 6 months after diagnosis or started radiotherapy more than 9 months after diagnosis, or they had insufficient information in their charts for analysis. For the present study, 924 of the remaining 1570 patients were excluded because they received curative radiotherapy as their primary treatment, leaving a final study sample of 646 patients who received radical prostatectomy as their primary treatment.

Medically trained data abstractors reviewed and abstracted patients' charts at their treating hospitals using a validated electronic chart abstraction tool. ${ }^{7}$ The study coordinator validated these abstractions in the field and also obtained and abstracted information from other sources (e.g., charts from secondary hospitals or general practitioners) when key data elements were missing from the treating charts. Diagnosis date, hospital volume, and vital status information from the CCE database were used, along with abstracted chart data for analysis. Vital status information was available up to December 31, 2007.

We selected 7 quality indicators from the published literature that were thought to influence survival and treatment-related morbidities. Based on feedback from clinical members of the research team, we also added pelvic lymph node dissection (PLND) during RP because it is increasingly being recognized as an aspect of care that may influence survival..$^{8,9}$ The candidate list of indicators included the following:

- hospital volume, the only structural indicator, which was defined as the average number of RPs performed at a single hospital per month;

- pre-treatment risk assessment, which included assessment of prostate-specific antigen (PSA) levels, digital rectal exam, clinical tumour stage, Gleason score, and prostate biopsy with at least 6 cores and the number of positive cores recorded;

- pre-treatment consultation with a radiation oncologist;

- appropriate follow-up care, defined as the number of years in which the recommended follow-up schedule was met (two follow-up visits with PSA testing in the first year, and one visit with PSA testing subsequently) divided by the number of years between surgery and the first occurrence of disease failure, chart abstraction date, or death;

- units of blood transfused during surgery and the postoperative period;

- use of a nerve-sparing surgical technique;

- surgical margin status; and

- PLND during RP.

Indicator-relevant outcomes were selected through an iterative process involving discussion and debate amongst the research team. This team included a urologist and a radiation oncologist, both of whom treat prostate cancer and were involved in the development of the published quality indicators lists used in this study. Indicator-relevant outcomes were selected based on the team's a priori hypotheses of what the component of care measured by the indicator was intended to achieve. One or two outcomes were selected for each indicator (see Appendix 1).

Long-term cause-specific survival was identified as a relevant outcome for hospital volume, pre-treatment risk assessment, follow-up care, surgical margin status, and PLND, and was defined as the time from surgery to death attributed to prostate cancer (International Classification of Disease [ICD]-9 185), with follow-up to the end of 2007. Diseasefree survival was selected as a relevant outcome for nervesparing surgery and operative blood loss. A disease-free survival event was defined as the earliest occurrence of local, regional, or distant failure, or death from prostate cancer, with follow-up limited to the date of last contact as per the chart abstraction data. We selected late morbidities, including erectile dysfunction and urinary incontinence more than 90 days after surgery, as a relevant outcome for operative blood loss, nerve sparing-sparing surgery, and surgical margin status. We identified a change in nodal status from clinical N0 to pathologic N1 following RP as a relevant outcome for pre-treatment assessment. We hypothesized that patients who did not receive the recommended assessments would be at increased risk of change in nodal stage, due to previously undetected nodal involvement. We chose surgical margin status (positive or negative) as the relevant outcome for pre-surgery consultation with a radiation oncologist, and we hypothesized that this consultation would be a marker for multidisciplinary management, which, in turn, would identify cases at higher risk of poor surgical outcomes, including positive margins. Appendix 1 outlines further details on the 
rationale behind our hypotheses.

We examined how these indicator-outcome associations were influenced by the following potential confounders: age at diagnosis; comorbidity, as measured by the Cumulative Illness Rating Scale for Geriatrics (Prostate) $\left(\text { CIRS- } \mathrm{G}_{(\text {pros })}\right)^{5}$; disease severity based on pre-treatment PSA, clinical tumour stage, and Gleason score; ${ }^{10}$ and treatment era.

\section{Statistical analysis}

Bivariate associations between quality indicators, outcomes, and potential confounders were examined with the Pearson Chi-square test, log rank test, or one-way analysis of variance (ANOVA). Both unadjusted and fully adjusted regressions were run for all indicators and their relevant outcomes. We used logistic regression to assess dichotomous outcomes and Cox proportional hazards regression to assess failure time outcomes. For the analyses of cause-specific survival, patients who died of other causes or who survived until the end of follow-up (December 31, 2007) were censored. For the analyses of disease-free survival, patients who died of other causes prior to or who were alive at the date of last contact in their medical charts were censored. We conducted a competing risks analysis using Kaplan-Meier survival curves and Cox proportional hazards regression to determine whether other-cause deaths might explain significant cause-specific survival results. ${ }^{11}$ All data processing and analyses were conducted using Statistical Analysis Software (SAS) version 9.2. ${ }^{12}$ Ethics approval for this study was obtained from the Queen's University Health Sciences Research Ethics Board.

\begin{tabular}{lcc}
\hline Table 1. Patient, disease, and treatment characteristics \\
\hline & N & $\%$ \\
\hline Age at diagnosis & & \\
$\quad<50$ & 14 & 2.2 \\
$50-54$ & 48 & 7.4 \\
$55-59$ & 110 & 17.0 \\
$60-64$ & 168 & 26.0 \\
$65-69$ & 220 & 34.1 \\
$70-74$ & 74 & 11.5 \\
$75+$ & 12 & 1.9 \\
Disease severity & & \\
$\quad$ Low & 235 & 42.3 \\
$\quad$ Intermediate & 237 & 42.6 \\
High & 84 & 15.1 \\
$\quad$ Unable to determine & 90 & \\
Treatment received & & \\
Surgery & 637 & 98.6 \\
Surgery and adjuvant radiotherapy & 9 & 1.4 \\
Surgical approach & & \\
$\quad$ Retropubic & 572 & 91.8 \\
Perineal & 50 & 8.0 \\
$\quad$ Suprapubic & 1 & 0.2 \\
Unknown & 23 & - \\
\hline
\end{tabular}

\section{Results}

The mean follow-up time was 58 months for time to last contact in the chart data and 132 months for survival data. Table 1 summarizes the age distribution, disease characteristics, and treatment details of the study sample. The patient characteristics reflect the fact that this is a surgically treated subset of prostate cancer patients, as their mean age was 63.1 years, which is lower than the average age at diagnosis for the general prostate cancer population (72-74 years), ${ }^{13-14}$ and over $80 \%$ had low or intermediate risk disease. Their mean CIRS- $\mathrm{G}_{\text {pros }}$ score was 2.1 ( $\mathrm{SD}=1.9$ ), indicates low comorbid illness; a score of 2 means either 2 mild current or significant past conditions or 1 moderately severe co-morbid condition. ${ }^{9}$ Almost half $(297 / 646 ; 46.0 \%)$ of the sample was diagnosed and treated from 1996 to 1998, with 233/646 (36.1\%) diagnosed and treated from 1993 to 1995 , and the remaining 116/646 (18.0\%) from 1990 to 1992.

Forty-five of the $646(7.1 \%)$ patients died of prostate cancer and 107/646 (16.8\%) died of other causes. One hundred and ten $(17.0 \%)$ patients experienced disease failure. The nodal stage of $12 / 646$ (1.9\%) patients changed from clinical N0 to pathologic N1, and 246 (43.5\%) of the 566 patients with known surgical margin status had positive surgical margins, although 220/566 (38.9\%) of those were minimally positive.

After examining data frequencies, we found that it was not feasible to assess the outcomes of urinary incontinence or erectile dysfunction because the information was too often missing from the charts (213/646 [33.0\%] and 376/646 [58.2\%] were missing, respectively). Ten patients were excluded from analyses of cause-specific and disease-free survival as they died prior to December 31, 2007, but were missing cause of death in the OCR.

We observed a statistically significant association between hospital volume and cause-specific survival $(p=0.05)$, with the gradient in the expected direction (Table 2, Fig. 1). After control for potential confounders, patients in the lowestvolume category were at greater risk of death from prostate cancer compared to those in the highest-volume category (HR 4.71; 95\% Cl 1.06-20.82, Table 2) while the results for patients in the middle 4 volume categories were not statistically significant. Table 3 presents the patient and disease characteristics by hospital-volume category. No association was observed between volume and patient age $(p=0.17)$, disease severity $(p=0.66)$, or comorbidity $(p=0.62)$. We conducted a competing risk analysis to assess whether death from causes other than prostate cancer explained the hospital-volume effect. We saw no opposing association with other-cause death, indicating that competing risk was not an explanation for our hospital-volume finding (results not shown). ${ }^{11}$ 
Table 2. Distributions and associations between quality indicators and their relevant outcomes

\begin{tabular}{|c|c|c|c|c|}
\hline & $\mathbf{n}$ & $\%$ & $\begin{array}{c}\text { Unadjusted HR } \\
(95 \% \mathrm{Cl})\end{array}$ & $\begin{array}{l}\text { Adjusted HR* } \\
\text { (95\% Cl) }\end{array}$ \\
\hline \multicolumn{5}{|l|}{ Hospital volume and cause-specific survival } \\
\hline$<1 \mathrm{RP} /$ month & 112 & 17.4 & $5.28(1.22-22.91)$ & $4.71(1.06-20.82)$ \\
\hline$<2 \mathrm{RP} /$ month & 164 & 25.5 & $2.76(0.61-12.46)$ & $2.51(0.54-11.58)$ \\
\hline$<3 \mathrm{RP} /$ month & 123 & 19.1 & $1.75(0.35-8.68)$ & $1.52(0.30-7.64)$ \\
\hline$<4 \mathrm{RP} /$ month & 104 & 16.2 & $2.22(0.43-11.48)$ & $2.51(0.48-13.14)$ \\
\hline$<7 \mathrm{RP} /$ month & 59 & 9.2 & $2.64(0.48-14.43)$ & $2.55(0.46-14.06)$ \\
\hline$\geq 7 \mathrm{RP} /$ month & 81 & 12.5 & 1.00 & 1.00 \\
\hline Missing & 3 & & $N / A$ & $\mathrm{~N} / \mathrm{A}$ \\
\hline \multicolumn{5}{|c|}{ Pre-treatment risk assessment and cause-specific survival } \\
\hline$\leq 2$ & 97 & 15.0 & $0.94(0.27-3.21)$ & $1.17(0.32-4.34)$ \\
\hline $3-4$ & 497 & 76.9 & $1.00(0.35-2.82)$ & $1.01(0.35-2.90)$ \\
\hline 5 & 52 & 8.1 & 1.00 & 1.00 \\
\hline \multicolumn{5}{|l|}{ Follow-up care and cause-specific survival } \\
\hline $0 \%$ & 205 & 31.7 & $0.28(0.12-0.65)$ & $0.37(0.15-0.94)$ \\
\hline$>0 \%-\leq 25 \%$ & 105 & 16.2 & $0.18(0.06-0.57)$ & $0.23(0.07-0.75)$ \\
\hline $25 \%-\leq 50 \%$ & 96 & 14.9 & $0.56(0.23-1.32)$ & $0.72(0.29-1.76)$ \\
\hline $50 \%-\leq 75 \%$ & 71 & 11.0 & $0.55(0.22-1.41)$ & $0.50(0.19-1.32)$ \\
\hline $75 \%-<100 \%$ & 88 & 13.6 & $0.20(0.06-0.69)$ & $0.24(0.07-0.87)$ \\
\hline $100 \%$ & 81 & 12.5 & 1.00 & 1.00 \\
\hline \multicolumn{5}{|l|}{ Nerve-sparing surgery and disease-free survival } \\
\hline No nerve-sparing surgery & 422 & 65.3 & $0.87(0.58-1.31)$ & $0.82(0.55-1.24)$ \\
\hline Unknown if nerve-sparing surgery used & 31 & 4.8 & $0.68(0.21-2.21)$ & $0.63(0.15-2.65)$ \\
\hline Nerve-sparing surgery used & 193 & 29.9 & 1.00 & 1.00 \\
\hline \multicolumn{5}{|l|}{ Operative blood loss and disease-free survival } \\
\hline$\geq 3$ units transfused & 157 & 24.3 & $1.28(0.55-1.41)$ & $1.44(0.91-2.26)$ \\
\hline 1-2 units transfused & 220 & 34.1 & $0.88(0.83-1.99)$ & $0.93(0.58-1.50)$ \\
\hline 0 units transfused & 269 & 41.6 & 1.00 & 1.00 \\
\hline \multicolumn{5}{|c|}{ Surgical margin status and cause-specific survival } \\
\hline Positive - extensive & 26 & 4.0 & $2.52(0.73-8.66)$ & $1.39(0.39-4.98)$ \\
\hline Positive - minimal & 220 & 34.1 & $1.47(0.74-2.94)$ & $1.05(0.51-2.18)$ \\
\hline Negative & 320 & 49.5 & 1.00 & 1.00 \\
\hline Unknown & 80 & 12.4 & $2.02(0.91-4.46)$ & $1.80(0.79-4.09)$ \\
\hline \multicolumn{5}{|c|}{ Pelvic lymph node dissection and cause-specific survival } \\
\hline No dissection & 116 & 18.0 & $1.00(0.44-2.24)$ & $1.71(0.73-4.01)$ \\
\hline \multirow[t]{2}{*}{ Dissection } & 530 & 82.0 & 1.00 & 1.00 \\
\hline & n & $\%$ & Unadjusted OR (95\% Cl) & Adjusted OR* $(95 \% \mathrm{Cl})$ \\
\hline \multicolumn{5}{|c|}{ Pre-treatment consultation with radiation oncologist and surgical margin status } \\
\hline No consultation & 573 & 88.7 & $1.30(0.77-2.20)$ & $1.30(0.75-2.26)$ \\
\hline Consultation & 73 & 11.3 & 1.00 & 1.00 \\
\hline \multicolumn{5}{|c|}{ Pre-treatment risk assessment and change from $\mathrm{cN} 0$ to $\mathrm{pN} 1$} \\
\hline$\leq 2$ & 97 & 15.0 & $1.07(0.10-12.13)$ & $1.87(0.14-24.47)$ \\
\hline 3-4 & 497 & 76.9 & $0.94(0.12-7.57)$ & $1.13(0.13-9.72)$ \\
\hline 5 & 52 & 8.1 & 1.00 & 1.00 \\
\hline
\end{tabular}

We also observed a statistically significant association between appropriate follow-up care and cause-specific survival $(p=0.03)$; there was no evidence of a gradient in the expected direction, however (Table 2, Fig. 2). Patients who received $0 \%, 0 \%$ to $\leq 25 \%$, and $75 \%$ to $<100 \%$ of the recom- mended follow-up visits were at a statistically significantly decreased risk of prostate cancer death compared to those who received $100 \%$ of the recommended follow-up visits (Table 2). We were concerned that the $100 \%$ follow-up care reference group may have had more severe disease than 
Webber et al.

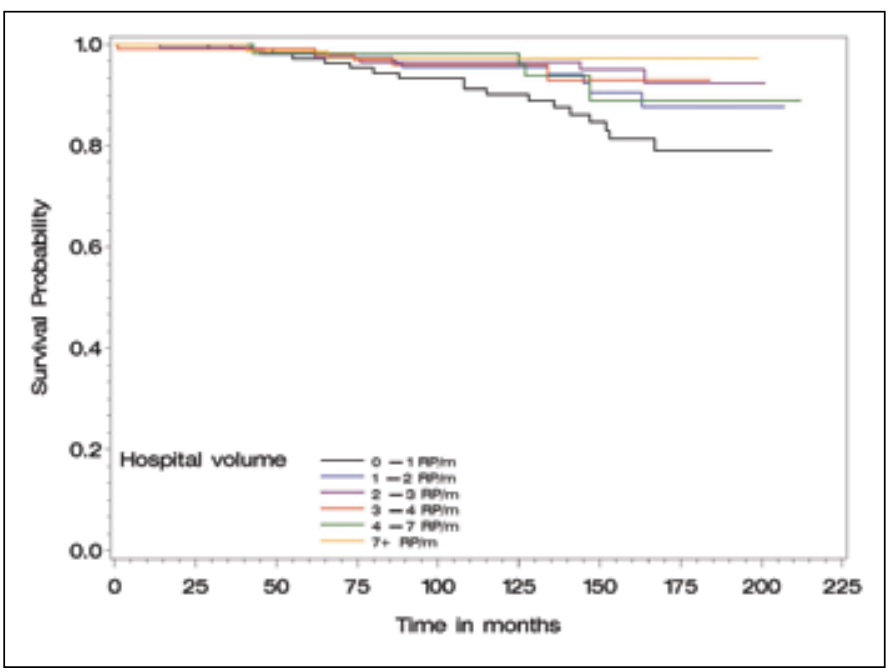

Fig. 1. The association between cause-specific survival and hospital volume.

could be represented in our data. In a secondary analysis, we chose a different reference group ( $75 \%$ to $<100 \%$ group) and found that patients who received $100 \%$ of the recommended follow-up care were at a significantly greater risk of prostate cancer death (HR 4.10, 95\% Cl 1.14-14.70), while patients in the remaining follow-up categories did not show a significant increased risk (results not shown). The competing risk of other-cause death does not explain the observed association between follow-up care and causespecific survival (results not shown).

None of the remaining quality indicators had a statistically significant association with their relevant indicators (Table 2). Of note, the effect estimates for the association between nerve-sparing surgery and disease failure were in the opposite direction to our original hypothesis, suggesting that non-nerve-sparing surgical techniques may decrease patients' risk of failure. We thought that this effect might have been due to an decreased risk of positive surgical margins in patients who received non-nerve-sparing surgery compared to those who received nerve-sparing surgery, but a secondary analysis of this association did not support this explanation (OR 1.14, 95\% Cl 0.81-1.62).

\section{Discussion}

Donabedian's quality of care model provides a useful framework upon which to base validation efforts. It requires an association between relevant outcomes and structure and process quality indicators such that high-quality care leads to increased likelihood of positive outcomes. ${ }^{4}$ To date, the quality indicators in our study have only been evaluated against intermediate outcomes, such as 30-day mortality and in-hospital complications. ${ }^{15-17}$ Our findings demonstrated that hospital volume can be validated through its association with an important long-term outcome in a population-based

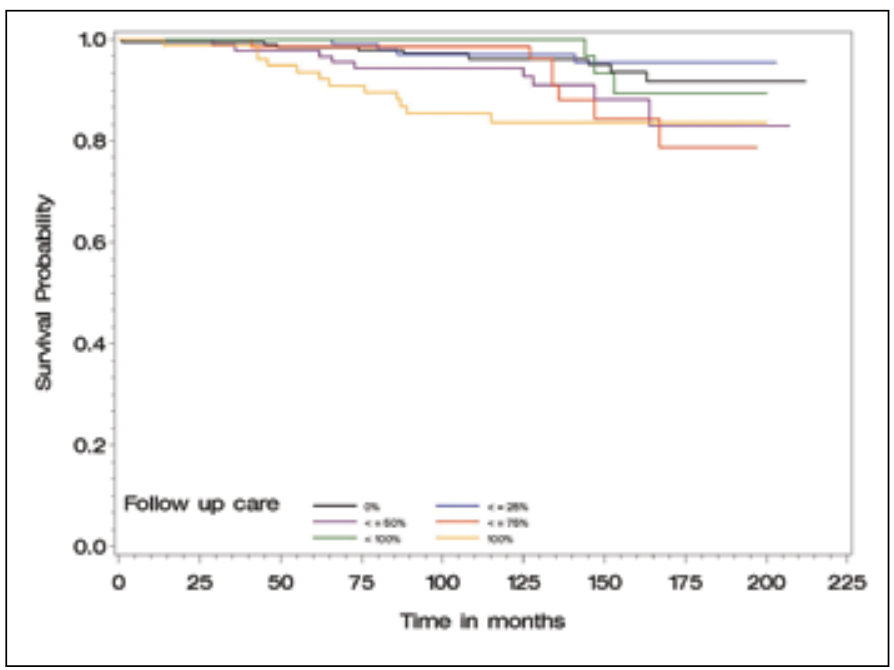

Fig. 2. The association between cause-specific survival and follow-up care.

sample of prostate cancer patients who received curative RP. Our negative findings for completeness of follow-up care suggest that it may only be possible to assess its validity in a prospective setting, due to its sensitivity to patient and disease status.

Some issues of statistical power hampered our validation efforts. Even with 17 years of follow-up, we only observed a $7 \%(n=45)$ prostate cancer-specific death rate. It was not feasible to increase our sample size because this study was part of a larger case-cohort study with province-wide chartbased data collected in the early 2000s. We did conduct our cause-specific survival analyses using the full case-cohort dataset, which contains information on $45 \%$ of the 187 prostate cancer deaths that occurred before 2000 in all surgically treated patients diagnosed in Ontario between 1990 and 1998. Those results yielded similar point estimates with a small increase in precision (results not shown). The statistical power issue that we faced is not unique to this study. Prostate cancer-specific survival is known to be challenging to study due to the long natural history of the disease, the rare nature of the outcome, and competing risks. ${ }^{18}$

Another challenge we faced in evaluating the validity of these quality of care indicators lies in the fact that many patients in our study received high-quality care. This is particularly notable considering that patients in our sample were diagnosed and treated years before these quality indicators were published and endorsed. This lack of variation in the quality of care received further limited our study power.

Several findings from this study warrant further discussion. The finding that surgery in a lower-volume hospital is associated with poorer long-term cause-specific survival is consistent with our a priori hypotheses and previous research, which has studied intermediate outcomes such as hospital readmission. ${ }^{15-26}$ This volume effect was not due to older patients or patients with more severe disease or comor- 


\begin{tabular}{|c|c|c|c|c|c|c|c|}
\hline & \multicolumn{6}{|c|}{ Hospital volume (RP/month) } & \multirow{2}{*}{$p$ value } \\
\hline & $<1$ & $<2$ & $<3$ & $<4$ & $<7$ & $\geq 7$ & \\
\hline Total $^{*}$ & 112 & 164 & 123 & 104 & 59 & 81 & \\
\hline \multicolumn{8}{|l|}{ Disease severity, n (\%) } \\
\hline Low & 35 (31.2) & $62(37.8)$ & $46(37.4)$ & $42(40.4)$ & $24(40.7)$ & $26(32.1)$ & \multirow{4}{*}{0.66} \\
\hline Intermediate & $41(36.6)$ & $57(34.8)$ & $38(30.9)$ & $40(38.5)$ & $23(39.0)$ & $38(46.9)$ & \\
\hline High & $16(14.3)$ & $23(14.0)$ & $19(15.4)$ & $10(9.6)$ & 7 (11.9) & $9(11.1)$ & \\
\hline Unknown & $20(17.9)$ & $22(13.4)$ & $20(16.3)$ & $12(11.5)$ & $5(8.5)$ & $8(9.9)$ & \\
\hline CIRS-G(pros) score, mean (SD) & $2.2(1.8)$ & $2.2(2.1)$ & $2.0(1.7)$ & $2.2(1.8)$ & $1.9(1.6)$ & $1.9(2.0)$ & 0.62 \\
\hline \multicolumn{8}{|l|}{ Age at diagnosis, $\mathrm{n}(\%)$} \\
\hline$<50$ & $3(2.7)$ & $2(1.2)$ & $5(4.1)$ & $3(2.9)$ & $0(0.0)$ & $1(1.2)$ & \multirow{7}{*}{0.17} \\
\hline $50-54$ & $7(6.2)$ & $13(7.9)$ & $8(6.5)$ & $11(10.6)$ & $3(5.1)$ & $6(7.4)$ & \\
\hline $55-59$ & $12(10.7)$ & 27 (16.5) & $24(19.5)$ & $20(19.2)$ & $9(15.3)$ & $18(22.2)$ & \\
\hline $60-64$ & $31(27.7)$ & $41(25.0)$ & $32(26.0)$ & $21(20.2)$ & $20(33.9)$ & $23(28.4)$ & \\
\hline $65-69$ & $35(31.2)$ & $60(36.6)$ & $40(32.5)$ & $35(33.6)$ & $19(32.2)$ & $29(35.8)$ & \\
\hline $70-74$ & $22(19.6)$ & 19 (11.6) & $10(8.1)$ & 12 (11.5) & $6(10.2)$ & $4(4.9)$ & \\
\hline $75+$ & $2(16.7)$ & $2(1.2)$ & $4(3.2)$ & 2 (1.9) & $2(3.4)$ & $0(0.0)$ & \\
\hline
\end{tabular}

*3 patients missing hospital volume assignment

bidities being referred to higher-volume centres. We are not aware of any other studies that have examined long-term survival following RP in relation to hospital volume. The observed association between follow-up care and survival was in the direction opposite to our a priori hypothesis, with patients who received poor follow-up care having better survival rates than those who received the recommended follow-up care. However, the relationship may make sense from a clinical standpoint, as physicians may provide more extensive follow-up care to patients who are at greater risk of poor outcomes. While we controlled for disease severity and comorbidity, we suspect that patients who received the recommended follow-up care had more severe disease than could be measured (and thereby controlled for) in our retrospective, chart-based data. Prospective studies are required to determine the purpose and impact of follow-up care in surgically treated prostate cancer patients.

The remaining 6 quality indicators that we studied did not show statistically significant associations with the selected outcomes and therefore firm conclusions cannot be made regarding their validity. While the effect estimates were generally in the hypothesized direction, with poor-quality care associated with an increased risk of prostate cancer death, one exception was nerve-sparing surgery. We had hypothesized that this surgery would be associated with a decreased risk of treatment-related morbidities while at the same time not affecting disease-free survival. We were unable to examine morbidities as an outcome, however, and our results suggest that not receiving nerve-sparing surgery may actually reduce the risk of disease recurrence. We found that this counterintuitive finding could not be explained by a lower risk of positive surgical margins in those who did not have nerve-sparing surgery. While it should again be emphasized that this result was not statistically significant, we think this association warrants further examination.

This study had several strengths. Both the chart and administrative data used in the analysis were of high quality. ${ }^{6,7,19}$ Trained data abstractors were blinded to the study hypotheses. The length of follow-up time for cause-specific survival was a further strength, because the impact of treatment on cause-specific survival does not typically appear until after 10 years, and our follow-up data ranged from 9 to 18 years. ${ }^{20}$

This study also had several limitations, aside from inadequate study power. There is potential misclassification in the measurement of quality indicators and outcomes due to our reliance on chart data. Specifically, misclassification may have occurred if care was given to patients but not documented in their charts. Our measurement of margin status may be hampered by lack of central pathology review. The available data did not allow us to examine all quality indicators and outcomes of interest. Our analysis of disease-free survival was limited by a shorter follow-up than was available for cause-specific survival. We used logistic regression for analyses of categorical outcomes, which estimates odds ratios, not relative risks. The odds ratio estimates from these analyses may overestimate the relative risk of the associations. Confounding by indication may be an issue in this study, as patients may have received high-quality care because physicians thought they were more likely to experience negative outcomes and thus were trying to prevent those outcomes.

\section{Conclusion}

This population-based study of 8 quality indicators generated several important findings. First, we demonstrated the validity of hospital volume through its association with 
Webber et al.

long-term survival, a relationship that has not been previously studied. Our findings call into question the validity of completeness of follow-up care as a quality indicator with a requirement of future prospective study of this indicator to better control for confounding by indication. The remaining 6 quality indicators that we studied were not significantly associated with the selected outcomes, and further study of the impact of these indicators on treatment-related morbidities is warranted. These outcomes are more common in this patient population and may be of greater concern to both physicians and patients.

Competing interests: Ms. Webber, Dr. Siemens, Dr. Brundage and Dr. Groome all declare no competing financial or personal interests.

This paper has been peer-reviewed.

\section{References}

1. Gagliardi AR, Fleshner N, Langer B, et al. Development of prostate cancer quality indicators: A modified Delphi approach. Can J Urol 2005;12:2808-15.

2. American Medical Association. Physician consortium for performance improvement 2008. http://www. ama-assn.org/apps/listserv/x-check/qmeasure.cgi? submit=PCPI. Accessed August 6, 2014.

3. Litwin MS, Steinberg M, Malin J, et al. Prostate cancer patient outcomes and choice of providers: Development of an infrastructure for quality assessment. Santa Monica: RAND Corporation; 2000.

4. Donabedian A. The quality of care: How can it be assessed? JAMA 1988;260:1743-8. http://dx.doi. org/10.1001/jama.1988.03410120089033

5. Groome PA, Rohland SL, Siemens DR, et al. Assessing the impact of comorbid illnesses on death within 10 years in prostate cancer treatment candidates. Cancer 2011;117:3943-52. http://dx.doi.org/10.1002/ cncr.25984

6. Mackillop W, Zhang-Salomons J, Groome PA, et al. Socioeconomic status and cancer survival in Ontario. J Clin Oncol 1997;15:1680-9.

7. Boulos DL. The contribution of comorbidity to survival outcomes in prostate cancer patients treated with curative intent. Queen's University; 2002.

8. Withrow DR, DeGroot JM, Siemens DR, et al. Therapeutic value of lymph node dissection at radical prostatectomy: A population-based case-cohort study. BJU Int 2011;108:209-16. http://dx.doi. org/10.1111/i.1464-410X.2010.09805.x

9. Bhatta-Dhar N, Reuther AM, Zippe C, et al. No difference in six-year biochemical failure rates with or without pelvic lymph node dissection during radical prostatectomy in low-risk patients with localized prostate cancer. Urology 2004;63:528-31. http://dx.doi.org/10.1016/i.urology.2003.09.064
10. D'Amico AV, Whittington $R$, Malkowicz SB, et al. Pretreatment nomogram for prostate-specific antigen recurrence after radical prostatectomy or external-beam radiation therapy for clinically localized prostate cancer. J Clin Oncol 1999;17:168-72.

11. Lau B, Cole SR, Gange SJ. Competing risk regression models for epidemiologic data. Am J Epidemiol 2009;170:244-56. http://dx.doi.org/10.1093/aje/kwp 107

12. SAS Institute. SAS (Statistical Analysis Software) Version 9.2; 2010.

13. Gronberg H. Prostate cancer epidemiology. Lancet 2003;361:859-64. http://dx.doi.org/10.1016/ S0140-6736(03) 12713-4

14. Goldenberg L, Ramsey EW, Jewett MAS. Prostate cancer 6: Surgical treatment of localized disease. Can Med Assoc J 1998; 159:1265-71.

15. Yao $S, L$ L-Yao GL. Population-based study of relationships between hospital volume of prostatectomies, patient outcomes, and length of hospital stay. J Natl Cancer Inst 1999;91:1950-6. http://dx.doi. org/10.1093/inci/91.22.1950

16. Barocas DA, Mitchell R, Chang SS, et al. Impact of surgeon and hospital volume on outcomes of radical prostatectomy. Urol Oncol: Semin Ori 2010;28:243-50. http://dx.doi.org/10.1016/i.urolonc.2009.03.001

17. Ellison $L M$, Heaney JA, Birkmeyer JD. The effect of hospital volume on mortality and resource use after radjcal prostatectomy. J Urology 2000;163:867-9. http://dx.doi.org/10.1016/S0022-5347(05)67821-4

18. Albertsen PC, Moore DF, Shih W, et al. Impact of comorbidity on survival among men with localized prostate cancer. J Clin Oncol 2011;29:1335-41. http://dx.doi.org/10.1200/JC0.2010.31.2330

19. Canadian Institute for Health Information. Data Quality Documentation: Discharge Abstract Database 2001-2002. Ottawa: Canadian Institute for Health Information; 2003.

20. Harwood RH. Management of localized prostate cancer: An epidemiological perspective. Clin Oncol ( $R$ Coll Radiol) 1995;7:151-9. http://dx.doi.org/10.1016/S0936-6555(05)80506-0

21. Potosky AL, Warren JL. Radical prostatectomy: Does higher volume lead to better quality? J Natl Cancer Inst 1999;91:1906-7. http://dx.doi.org/10.1093/inci/91.22.1906

22. Malkowicz SB, Vaughn DJ, Wein AJ. Adult genitourinary cancer: Prostate and bladder. In: Hanno PM, Malkowicz SB, Wein AJ, editors. Penn Clinical Manual of Urology. Philadelphia: Saunders Elsevier; 2007:523-69. httrp://dx.doi.org/10.1016/B978-141603848-1.10015-4

23. Litwin MS, Steinberg M, Malin J, et al. Prostate cancer patient outcomes and choice of providers: Development of an infrastructure for quality assessment. Santa Monica, CA: RAND; 2000.

24. Chan EOM, Groome PA, Siemens DR. Validation of quality indicators for radical prostatectomy. Int I Cancer 2008;123:2651-7. http://dx.doi.org/10.1002/iic.23782

25. Graefen M, Walz J, Huland H. Open retropubic nerve-sparing radical prostatectomy. Eur Urol 2007;49:3848. http://dx.doi.org/10.1016/j.eururo.2005.10.008

26. Yossepowitch 0 , Briganti A, Eastham JA, et al. Positive surgical margins after radical prostatectomy: A systematic review and contemporary update. Eur Urol 2014;65:303-13. http://dx.doi.org/10.1016/i. eururo.2013.07.039

Correspondence: Dr. Patti A. Groome, Division of Cancer Care and Epidemiology, Queen's Cancer Research Institute, Queen's University, Kingston, ON; groomep@queensu.ca 


\section{Appendix 1. Hypothesized associations between quality indicators and outcomes}

Quality indicator Outcomes Hypothesized association and rationale

Hospital volume is thought to be a proxy measure for the expertise of treating physicians within a hospital. Surgeons working in high-volume hospitals have greater experience treating

Hospital volume Cause-specific patients and thus more opportunities to refine their skills, while surgeons working in lowsurvival volume hospitals will not have these opportunities. ${ }^{21}$ Thus, it is hypothesized that patients treated in high-volume hospitals will have longer cause-specific survival than patients treated in low-volume hospitals. As hospital volume increases, the cause-specific survival of patients will also increase.

$\begin{array}{ll}\text { Appropriate pre- } & \text { Cause-specific } \\ \text { treatment risk } & \text { survival, pathologic } \\ \text { assessment } & \begin{array}{l}\text { stage after radical } \\ \text { prostatectomy }\end{array}\end{array}$

Pre-surgery consultation with a radiation oncologist

Margin status in surgical patients

Pre-surgery consultation with a radiation oncologist

Margin status in surgical patients

$\begin{array}{ll}\text { Appropriate } & \text { Cause-specific } \\ \text { follow-up care } & \text { survival }\end{array}$

\section{Appropriate} survival

Operative blood loss

Disease-free survival, late morbidity
The goal of risk assessment prior to treatment is to determine if a patient is an appropriate candidate for curative treatment and what treatment is most appropriate. ${ }^{22}$ Without an appropriate risk assessment, it is possible that patients' disease will be under-staged and their risk level underestimated, and thus treatment will not be aggressive enough. Thus, it is hypothesized that patients with inappropriate pre-treatment risk assessment will have shorter cause-specific survival compared to patients with appropriate risk assessment.

The goal of multidisciplinary care in the treatment of prostate cancer is to ensure that patients receive appropriate curative treatment. ${ }^{23}$ For some patients, surgery is not an appropriate treatment option, as their cancer has spread outside the prostate capsule and is thus not operable. A consultation with a radiation oncologist prior to surgery is intended to identify patients who are not good surgical candidates and who therefore may experience adverse outcomes following surgery, including positive surgical margins. Thus, it is hypothesized that patients who do not receive a consultation with a radiation oncologist will be more likely to have positive margins after surgery than those who do receive a consultation.

The goal of multidisciplinary care in the treatment of prostate cancer is to ensure that patients receive appropriate curative treatment. ${ }^{23}$ For some patients, surgery is not an appropriate treatment option, as their cancer has spread outside the prostate capsule and is thus not operable. A consultation with a radiation oncologist prior to surgery is intended to identify patients who are not good surgical candidates and who therefore may experience adverse outcomes following surgery, including positive surgical margins. Thus, it is hypothesized that patients who do not receive a consultation with a radiation oncologist will be more likely to have positive margins after surgery than those who do receive a consultation.

The goal of follow-up care is to monitor patients for disease recurrence and allow for early treatment of recurring cancer. ${ }^{22}$ The earlier disease recurrences are treated, the better the patients' long-term survival. Thus, it is hypothesized that patients who receive appropriate follow-up care will have longer cause-specific survival than patients who have received inadequate follow-up care.

Blood loss during radical prostatectomy is thought to be a proxy measure for the quality of the surgical technique used. For the proposed study, units of blood transfused during surgery will be used as a measure of operative blood loss. While transfusion of 1 or 2 units of blood is anticipated during radical prostatectomy (RP), transfusion of 3 or more units is thought to be excessive and indicative of low-quality surgical techniques. ${ }^{24}$ Patients who receive poor-quality surgery are thought to have poorer outcomes than those who receive high-quality surgery. Thus, it is hypothesized that patients who receive 3 or more units of blood during surgery will have shorter time to recurrence (disease-free survival) than those who receive only 1 or 2 units of blood. Furthermore, patients who receive 3 or more units of blood during RP will have more late morbidities than those who receive only 1 or 2 units of blood.

Nerve-sparing surgery is intended to preserve the nerves serving the organs and tissues surrounding the prostate. In particular, these nerves promote erectile function and bladder continence. ${ }^{25}$ The use of nerve-sparing techniques during RP is thought to lessen morbidities after treatment while not affecting patients' survival. Thus, it is hypothesized that patients who receive nerve-sparing radical prostatectomy will have fewer late morbidities than those patients who receive non-nerve-sparing surgery. However, it is hypothesized that the diseasefree survival will be the same for those patients who receive nerve-sparing surgery and those that receive non-nerve-sparing surgery.

As with operative blood loss, positive margins after radical prostatectomy are thought to be a proxy measure for the quality of the surgical technique used. ${ }^{26}$ Patients with positive margins following radical prostatectomy are thought to have received poorer-quality surgery than patients with negative margins following surgery. Therefore, it is hypothesized that patients who have positive margins following RP will have shorter cause-specific survival than those with negative margins, and that patients with positive margins following surgery will have greater late morbidities following RP than those with negative margins. 